\title{
Etude sur les corrélations entre le standard penetration test et le pressiomètre
}

\section{Correlation study between standard penetration and pressuremeter tests}

\author{
H. GONIN \\ Ingénieur ENPC* \\ P. VANDANGEON \\ Ingénieur ENSG* \\ Marie-Pierre LAFEUILLADE \\ Ingénieur IST*
}

Rev, Franç. Géotech. n 58, pp. 67-78 (janvier 1992)

\section{Résumé}

Les auteurs, se basant sur le mémoire de fin d'études de l'un d'eux, présentent une étude de corrélations entre valeurs des essais de pénétration au carottier (SPTN) et essais pressiométriques (pression limite PI et module E). Neuf formations argileuses, sableuses, marneuses et crayeuses de la région parisienne et du Nord de la France ont été étudiées. L'article comporte dans une première partie une synthèse bibliographique, et dans une deuxième partie les résultats de l'étude statistique effectuée.

En conclusion, quelques réflexions sont présentées sur la représentativité des essais et l'utilisation de corrélations pour apprécier les tassements.

\section{Abstract}

A correlation study between SPT and pressuremeter test is evaluated by the authors. Nine different formations, including clays, sands, marls and chalk from the Paris and North of France sedimentary basin have been considered. The article outlines initially a litterature review on the subject and further considerates statistical relationships between both tests.

As a conclusion, a discussion on the representativity of the tests and the use of these correlations to predict settlements are presented. 


\section{PRÉSENTATION}

Le «Standard Penetration Test » (SPT) est un essai de pénétration dynamique qui permet de tester la résistance du sol tout en prélevant des échantillons remaniés du terrain. Il est utilisé de manière extensive dans le monde entier. En France, cependant, l'essai pressiométrique MENARD lui est souvent préféré et notre réglementation (DTU, fascicule 62) se réfère de manière préférentielle aux valeurs pressiométriques.

Aussi, apparaît-il nécessaire de comparer les résultats obtenus entre ces deux types d'essais dans les mêmes formations géologiques et d'étudier la possibilité de corréler ces résultats.

Cette démarche a été le but du mémoire présenté et soutenu le 19 octobre 1990 par M.-P. COVILLELAFEUILLADE en vue de l'obtention du diplôme d'ingénieur en géophysique et géotechnique de l'Institut des sciences et technologie de l'Université Pierre et Marie Curie, Jussieu Paris VI. L'auteur s'est appuyé sur les très nombreux résultats d'essais pressiométriques et d'essais Standard Penetration Test effectués dans des formations géologiques caractéristiques de la région parisienne et du Nord de la France pour déterminer les graphiques donnant pour chaque formation étudiée la relation entre ces résultats. Son étude a impliqué le choix d'une méthode rigoureuse pour l'exploitation statistique de l'ensemble des données, permettant que les valeurs retenues le soient à partir de critères objectifs.

On se propose ici de reprendre l'essentiel des résultats obtenus afin de valoriser chacun de ces essais par les corrélations les reliant l'un à l'autre; elle porte sur neuf formations géologiques recouvrant la grande majorité de types de sols rencontrés dans la nature : sables, limons, argiles, marnes, craie.

De nombreux auteurs s'étant antérieurement penchés sur les corrélations pouvant être trouvées entre le SPT et différents paramètres caractérisant les sols, il a semblé intéressant, avant d'aborder l'étude se rapportant aux corrélations SPT/pressiomètre, de présenter une synthèse des publications concernant ces autres corrélations, si bien que cet exposé se décomposera en deux parties :

- une première partie se rapportant à la présentation générale du SPT et à la synthèse bibliographique des résultats et corrélations obtenues;

- une deuxième partie se rapportant à la présentation de l'étude comparative des résultats des essais SPT et des essais pressiométriques et aux corrélations qui s'en déduisent.

\section{RAPPELS ET SYNTHÈSE BIBLIOGRAPHIQUE SUR LE SPT}

L'essai SPT (Standard Penetration Test) vient des Etats-Unis; il a été largement développé par TERZAGHI qui a défini un mode opératoire pour ce qui n'était, au début, qu'une habitude de chantier. Il s'agit en effet de compter le nombre de coups nécessaire pour enfoncer un carottier normalisé sur $0,30 \mathrm{~m}$ de profondeur avec une masse de $63,5 \mathrm{~kg}$ tombant en chute libre d'une hauteur de $0,76 \mathrm{~m}$.

Ce nombre de coups correspond à la valeur * SPT », $\mathrm{N}$ suivant la Norme ASTM, le projet de Norme européenne publié dans les Proceedings du Congrès International de mécanique des sols à Tokyo en 1981 .

Cet essai, qualifié parfois de rustique, donne cependant des résultats raisonnablement fidèles et présente deux avantages peu souvent mis en lumière:

- fait à l'avancement, il est moins sensible que le pressiomètre à la qualité du trou de forage ;

- il est le seul essai qui associe, à une mesure in situ de la résistance du sol, un prélèvement permettant une identification précise des terrains soumis à l'essai.

Les paramètres, données ou grandeurs physiques, qui ont été le plus souvent évalués à partir des valeurs de l'essai SPT, sont les suivants :

- densité relative du sable ;

- angle de frottement interne d'un sable;

- résistance à la compression simple d'une argile ;

- contrainte admissible sur un sable ;

- potentiel de liquéfaction d'un sol ;

- pression de pointe mesurée au «pénétromètre statique $» ;$

- pression limite et module pressiométrique mesurê au pressiomètre MENARD.

Nous nous contentons ici de récapituler les correspondances proposées, sans porter de jugement sur leur validité.

\subsection{Densité relative d'un sable}

La densité relative d'un sable est définie par l'une des relations suivantes:

$$
\begin{gathered}
\operatorname{Dr}=\frac{e_{\max }-e}{e_{\max }-e_{\min }} \times 100 \\
\operatorname{Dr}=\frac{\gamma_{\max }}{\gamma_{d}} \times \frac{\gamma_{d}-\gamma_{\min }}{\gamma_{\max }-\gamma_{\min }} \times 100
\end{gathered}
$$

où " $e$ » est l'indice des vides et « $\gamma$ »le poids volumique sec du sable.

TERZAGHI et PECK ont donné, (fig. 1) une correspondance entre la densité relative d'un sable et sa résistance mesurée au SPT.

Pour corréler ces deux valeurs, SCHULTZ et MENZENBACK, tenant compte de la contrainte effective du sol au niveau de l'essai, préfèrent utiliser la relation suivante:

$\operatorname{Ln}(\mathrm{Dr})=0,478 \operatorname{Ln}(\mathrm{N})-0,262 \operatorname{Ln}\left(\sigma^{\prime}{ }_{\mathrm{vo}}\right)+2,84$

Ln : logarithme népérien ;

$\sigma_{\text {vo }}^{\prime}:$ contrainte effective au repos exprimée en $10^{5}$ $\mathrm{Pa}$ (bar). 


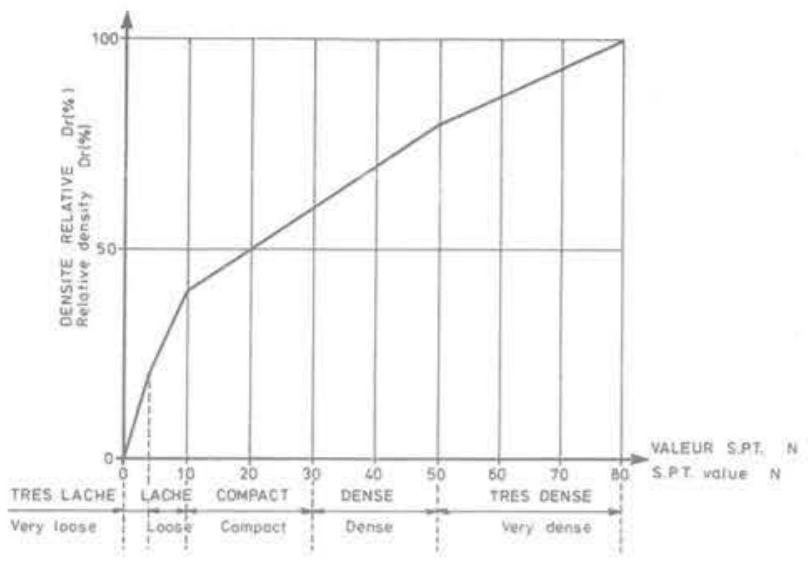

Fig. 1. - Correspondance entre $N$ et la densité relative $D_{r}$ d'un sable.

Fig. 1. - Relationship between $\mathrm{N}$ and relative density $D_{r}$ for sand.

\subsection{Angle de frottement interne d'un sable}

Plusieurs auteurs ont essayé de trouver une relation entre l'angle de frottement et la résistance d'un sable mesuré au SPT.

MEYERHOF donne deux expressions approchées de l'angle de frottement interne d'un sable en fonction de sa densité relative, selon le pourcentage de sable fin et de silt.

$\varphi=25+0,15 \mathrm{D}_{r}$,

(plus de $5 \%$ de sable fin et de silt);

$\varphi=35+0,15 D_{r}$,

(moins de $5 \%$ de sable et de silt) ;

( $\varphi$ en degrés)

relations expérimentales et empiriques qui ont été mises sous forme d'un abaque permettant de relier directement l'angle de frottement $\varphi$ à la valeur SPTN à partir des résultats d'essais en laboratoire effectués sur les échantillons prélevés par le carottier SPT luimême (fig. 2).

Signalons deux autres formules existant pour exprimer la relation entre $\varphi$ et $N$ :

$$
\begin{array}{lll}
\varphi=\sqrt{12 \mathrm{~N}}+25 & & \text { (DUNHAM) } \\
\varphi=\sqrt{20 \mathrm{~N}}+15 & & \text { (OSAKI) }
\end{array}
$$

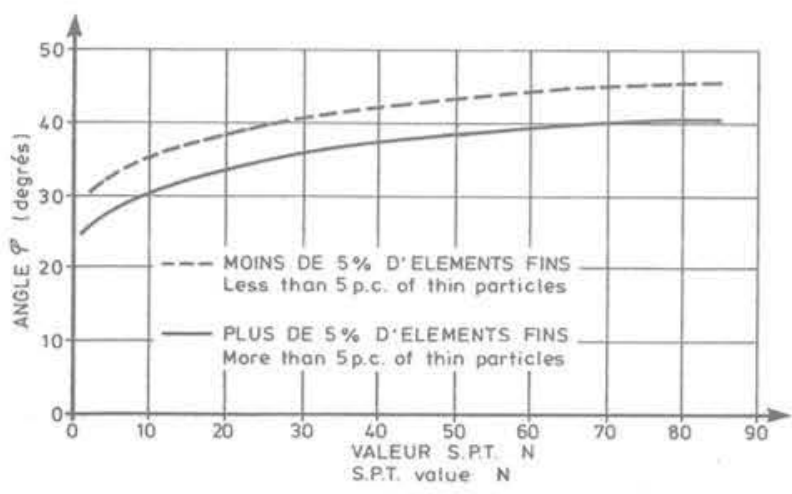

Fig. 2. - Corrélation entre $N$ et angle de frottement $\varphi$. Fig. 2. - Correlation between $N$ and friction angle $\varphi$.
Les valeurs de $\varphi$ en degrés déterminées à partir de ces abaques et formules sont approximatives, mais conduisent à un ordre de grandeur raisonnable.

\subsection{Résistance à la compression simple d'une argile}

Suivant la qualité d'une argile (molle à dure), TERZAGHI et PECK ont donné des corrélations entre la valeur SPT, $\mathrm{N}$, et la cohésion non drainée $\mathrm{Cu}$, transcrites dans le tableau I.
Tableau I. - Relation entre $\mathrm{N}$ et $\mathrm{Cu}$ pour les argiles (d'après TERZAGHI et PECK).

Table I. - Relationship between $N$ and $\mathrm{Cu}$ for clays (TERZAGHI et PECK).

\begin{tabular}{|l|c|c|}
\hline $\begin{array}{c}\text { Consistance } \\
\text { des argiles }\end{array}$ & Valeur STPN & $\begin{array}{c}\text { Cohésion } \\
\text { non drainée } \\
\text { Cu (kPa) }\end{array}$ \\
\hline Très molle & $<2$ & $<12,5$ \\
Molle & 2 à 4 & 12,5 à 25 \\
Plastique & 4 à 8 & 25 à 50 \\
Ferme & 8 à 15 & 50 à 100 \\
Très ferme & 15 à 30 & 100 à 200 \\
Dure & $>30$ & $>200$ \\
\hline
\end{tabular}

Ces mêmes auteurs ont également donné les relations suivantes $(\mathrm{Cu}$ en $\mathrm{kPa})$ :

$$
\begin{array}{ll}
\text { - argiles plastiques } & \mathrm{Cu}=12,5 \mathrm{~N} \\
\text { - argiles silteuses } & \mathrm{Cu}=10 \mathrm{~N} \\
\text { - argiles sableuses } & \mathrm{Cu}=6,7 \mathrm{~N}
\end{array}
$$

Il s'agit là aussi de valeurs approximatives. En fait, les relations données par TERZAGHI et PECK se réfèrent à la résistance à la compression simple, prise égale à $2 \mathrm{Cu}$ dans le tableau I et les formules présentées ci-dessus.

\subsection{Contrainte admissible sur du sable}

D'une manière générale, aux Etats-Unis, les contraintes admissibles pour des semelles fondées sur du sable sont déterminées à l'aide des abaques des figures 3 et 4 qui tiennent compte d'un coefficient de sécurité de 3 par rapport à la rupture.

Ces abaques ne sont applicables que si le niveau de la nappe phréatique est au moins à une profondeur B (largeur de la semelle) au-dessous de la base des semelles ; dans le cas contraire, les pressions admissibles doivent être divisées par un coefficient réducteur compris entre 1 et 2 .

En admettant un critère de tassement maximum de 1 pouce $(2,54 \mathrm{~cm})$, l'abaque de la figure 5 permet de déterminer la contrainte admissible.

\subsection{Potentiel de liquéfaction d'un sol}

Les premières tentatives d'évaluation de la liquéfaction d'un sol par la valeur SPT $\mathrm{N}$ datent du séisme de Niigata en 1964, séisme de magnitude 7,5. Depuis cette époque, de nombreux travaux ont complété cette recherche. 


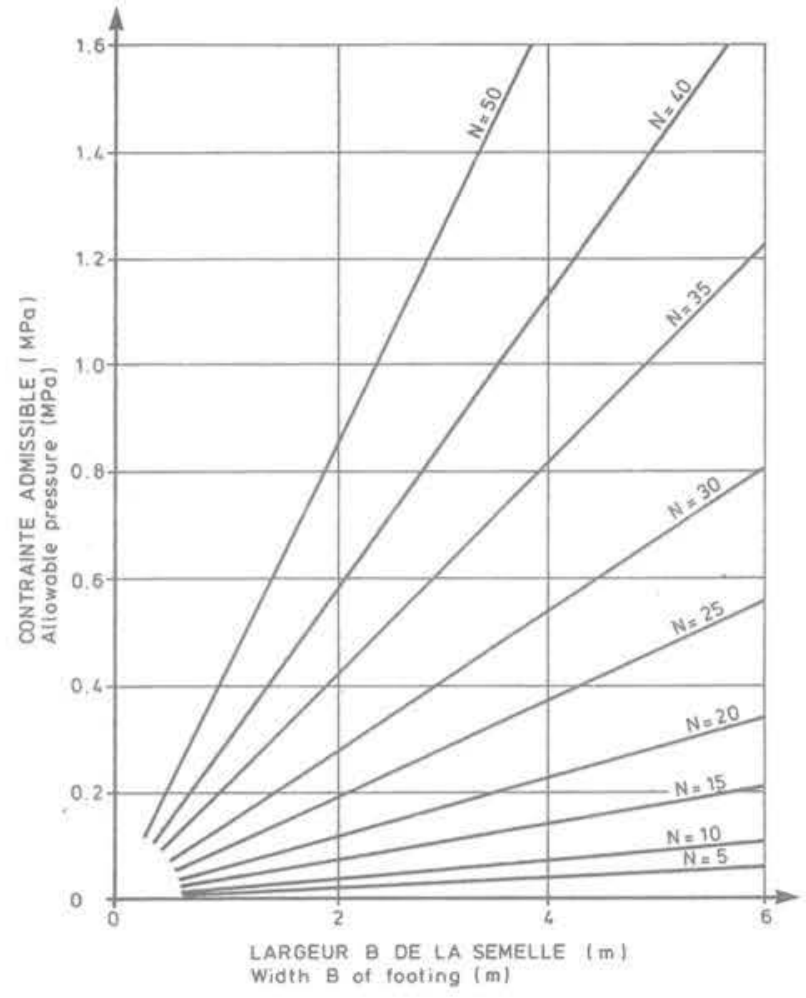

Fig. 3. - Contrainte admissible en surface.

Fig. 3. - Allowable pressure without leteral loading.

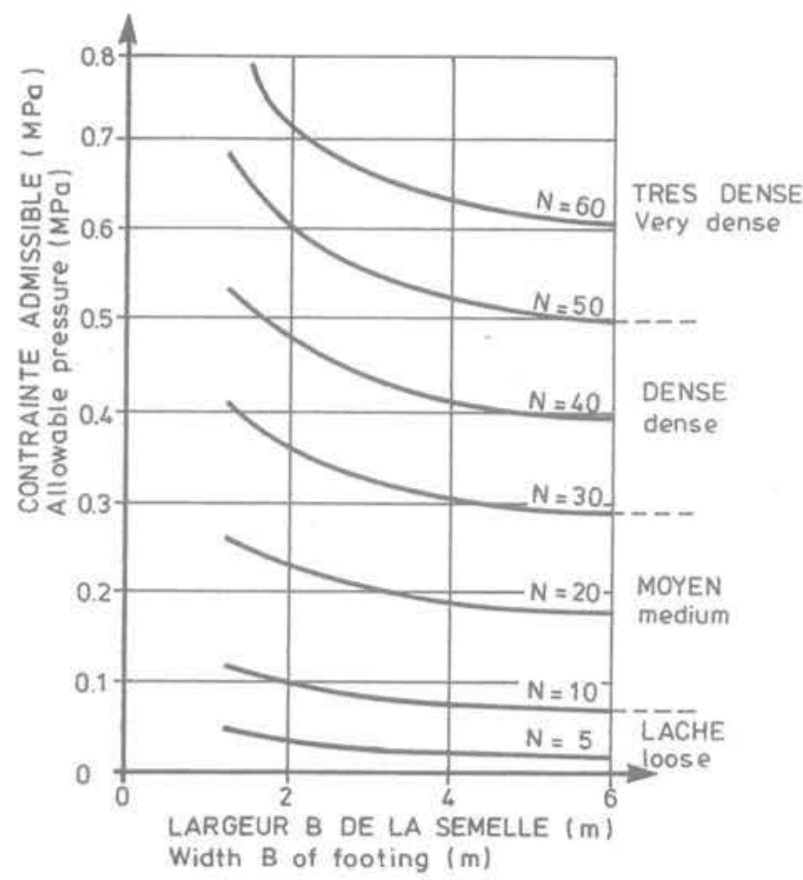

Fig. 5. - Relation entre $n$, la largeur de la semelle et la contrainte admissible.

Fig. 5. - Relationship between N, footing width and allowable admissible pressure.

L'étude de plus de trente cinq cas a permis à SEED, MORI et CHAN d'exprimer le rapport des contraintes $\tau \mathrm{av} / \sigma^{\prime} \mathrm{v}$ en fonction d'une valeur corrigée de la résistance à la pénétration.

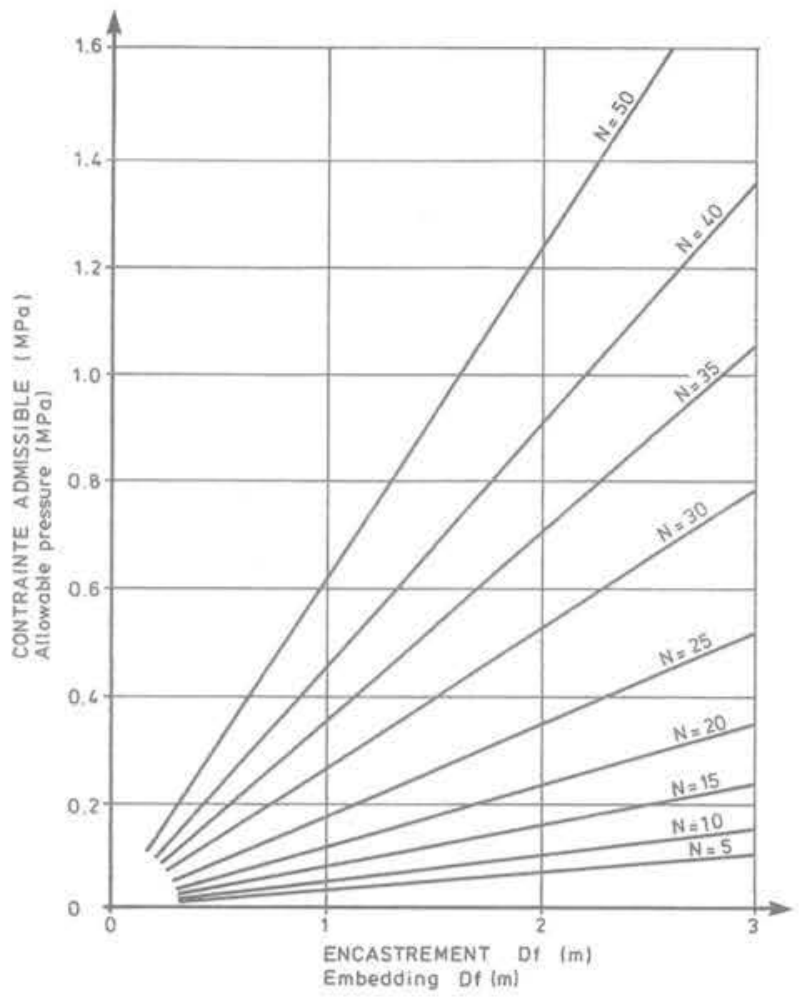

Fig. 4. - Accroissement de la contrainte admissible do à l'encastrement Df.

Fig. 4. - Increase of allowable pressure due to embedding Df.

$\sigma^{\prime} v$ étant la contrainte verticale effective à la profondeur considérée :

Tav étant la contrainte moyenne de cisaillement due au séisme à cette profondeur.

La valeur SPT, $N$, est modifiée pour se ramener à une contrainte verticale effective de 1 ton/sq.ft. (1,076 bar). La valeur corrigée $\mathrm{N}_{1}$ est ainsi reliée à $\mathrm{N}$ par la relation $\mathrm{N}_{1}=\mathrm{C}_{\mathrm{n}} \times \mathrm{N}_{1}$ le coefficient $\mathrm{C}_{\mathrm{n}}$ étant donné par la figure 6 .

La corrélation $\tau_{a y} / \sigma_{v}^{\prime} / \mathrm{N}_{1}$ est représentée sur la figure 7 avec la délimitation du domaine contenant l'ensemble des sites où la liquéfaction a été observée. Cette limite est représentée par la droite

$$
\tau_{\text {av }} / \sigma_{v}=\mathrm{N}_{1} / 90 \text { pour } \mathrm{N}_{1}<35 \text {. }
$$

Il est à noter que la proportion d'éléments fins dans le sol influe sur les corrélations reliant la liquéfaction du sol à sa résitance SPT. En effet, la liquéfaction touche essentiellement les sols sans cohésion. Cela se traduit par le fait qu'à valeur $\mathrm{N}$ égale, plus un sol est riche en particules fines, plus il résiste au cisaillement, donc à la liquéfaction

\subsection{Résistance en pointe mesurée au pénétromètre statique (CPT)}

Un nombre important de travaux comparant les valeurs $\mathrm{N}$ et la résistance à la pénétration statique $q_{c}$ ont été publiés, conduisant à des variations assez larges des valeurs du rapport $q_{c} / N$. Une des raisons 


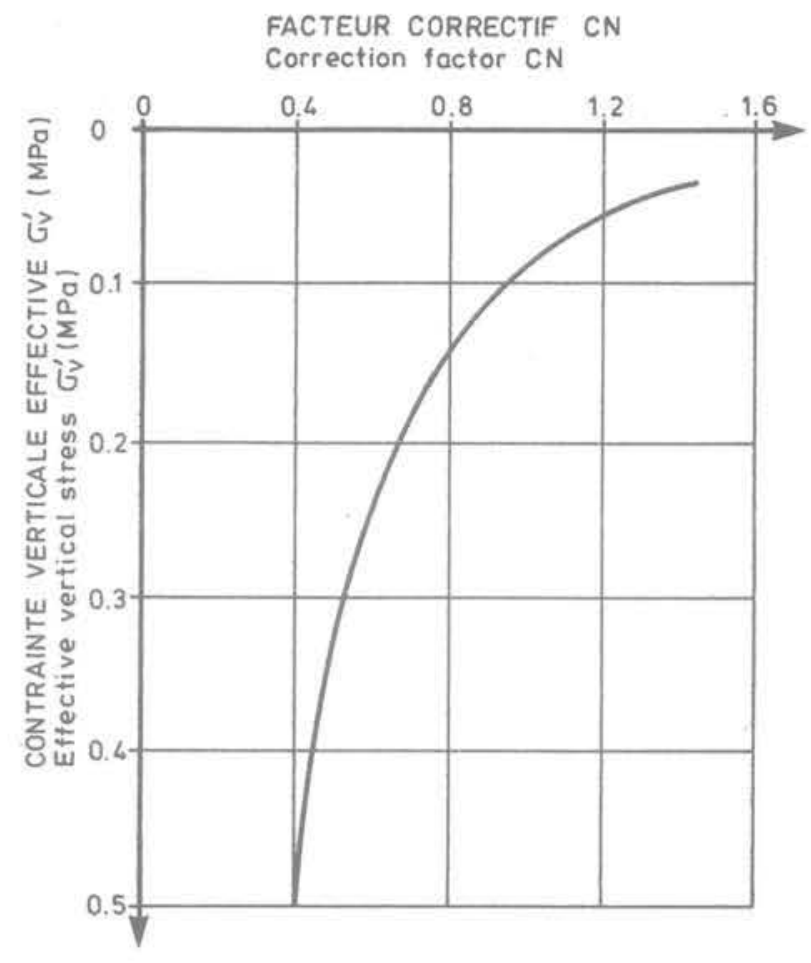

Fig. 6. - Coefficient correcteur $C_{N}$. Fig, 6. - Correction factor $C_{N}$.

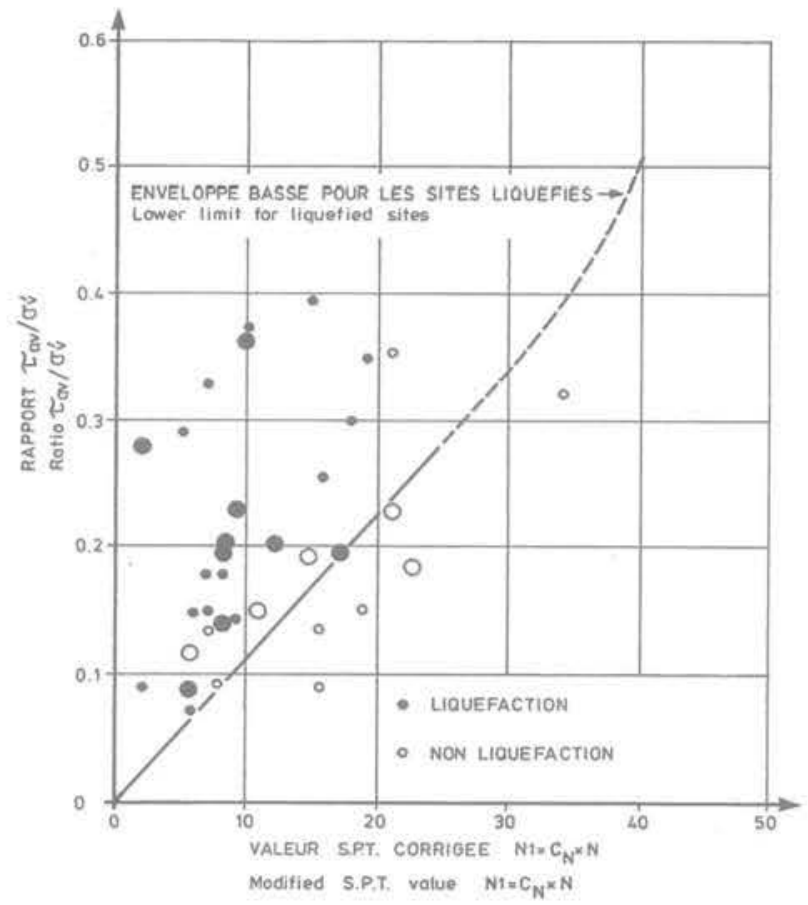

Fig. 7. - Relation entre $N$ et le comportement du site pour la liquéfaction.

Fig. 7. - Relationship between $N$ and site behaviour for liquefaction.

objectives de cet éventail tient à l'influence de la taille des grains. La figure 8 montre clairement que le rapport $\mathrm{q}_{\mathrm{c}} / \mathrm{N}$ augmente avec le diamètre moyen $\mathrm{D}_{50}$ des grains.

La synthèse des corrélations données par différents auteurs conduit aux résultats ci-après $\left(\mathrm{q}_{\mathrm{c}}\right.$ en $\left.\mathrm{MPa}\right)$ :

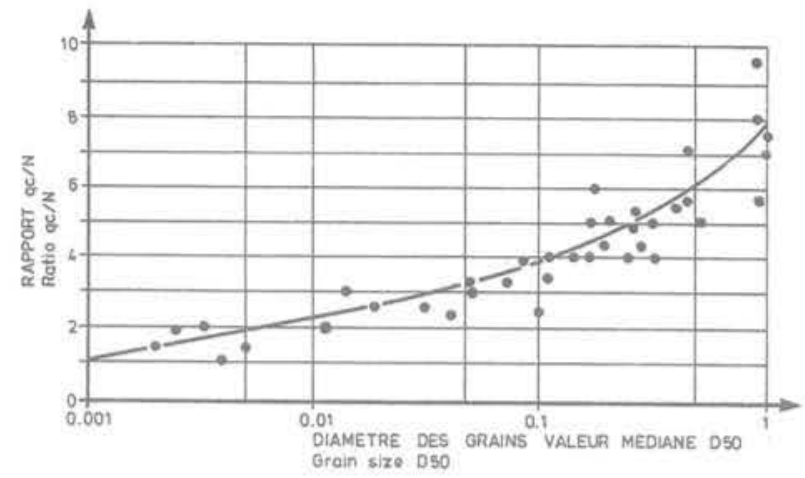

Fig. 8. - Corrélation SPT-CPT : influence de la taille des grains. Fig. 8. - Correlation SPT-CPT: grain size influence.

- sols à dominance sableuse : $\mathrm{q}_{\mathrm{c}}=0,2 \times \mathrm{N}$ à $0,6 \times \mathrm{N}$

- sols à dominance argileuse : $\mathrm{q}_{\mathrm{c}}=0.1 \times \mathrm{N}$ à $0,4 \mathrm{~N}$.

La correspondance la plus couramment trouvée et utilisée quelle que soit la nature du terrain est :

$$
\mathrm{q}_{\mathrm{c}}=0,2 \times \mathrm{N}
$$

\subsection{Pression limite et module mesuré au pressiomètre MENARD}

Les résultats étudiés et présentés à ce jour sont très fragmentaires, car le nombre d'essais comparatifs est assez restreint du fait des habitudes nationales privilégiant tel type d'essai au détriment de tel autre et également, parce que chaque type d'essai a son terrain d'élection.

C'est une des raisons ayant conduit Marie-Pierre LAFEUILLADE à tenter de combler cette carence en utilisant le grand nombre d'essais SPT effectués en France dans les études faites ou suivies par SIMECSOL depuis plus de trente ans.

Concernant les résultats fragmentaires donnés à ce jour, ils sont en majeure partie repris par CASSAN dans son ouvrage "Essais in situ en mécanique des sols " (fig. 9).

Ils conduisent aux relations suivantes :

- pour les argiles de Nice : $\mathrm{N}=10$ à $30(\mathrm{Pl}$. $\left.\mathrm{P}_{0}\right)$;

- pour les sols du Barcarès : $N$ \# $50\left(\mathrm{Pl}-\mathrm{P}_{0}\right)$; - pour les sols de Dunkerque : $N \# 6 \times E$.

Cette comparaison entre Standard Penetration Test et pressiomètre est en fait l'objet de la présente étude ; nous ne nous étendrons donc pas sur ces premiers résultats qui, ainsi que nous le verrons, sont dans l'ensemble conformes aux résultats analysés dans la $2^{e}$ partie de cette étude.

\section{MÉTHODOLOGİE DE L'ÉTUDE}

\subsection{Les limites de l'étude}

Cette étude sur les corrélations entre les paramètres pressiométriques et SPT a été réalisée statistiquement sur un ensemble de mille six cent dix mesures. Une unité de profondeur, de situation géographique et de formation géologique a été respectée. C'est-à-dire que la comparaison n'a porté que sur des essais effectués 


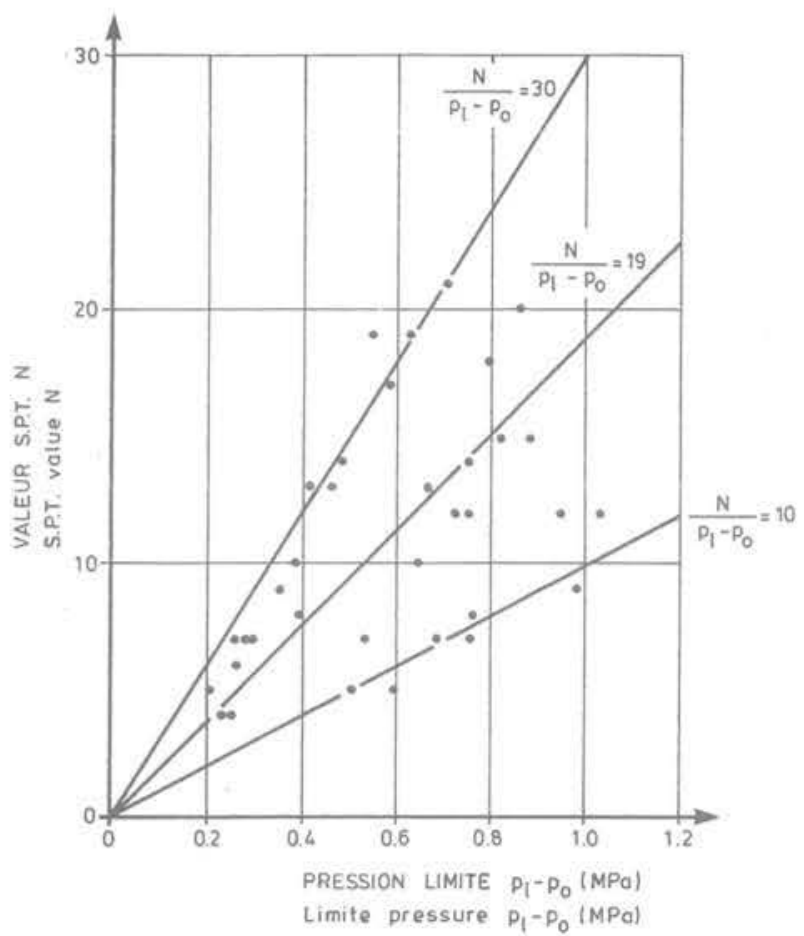

Fig. 9. - Corrélation entre $N$ et Pl-Po en terrain cohérent. Fig. 9. - Correlation between N and PI-PO for coohesive soils.

à une même profondeur, dans une même formation et pour des sondages distants de moins d'une trentaine de mètres l'un de l'autre en plan et à des altitudes comparables.
Les principales formations géologiques qui ont pu ainsi être étudiées grâce à un nombre suffisant d'essais sont les suivantes :

- limons bruns des plateaux de la région parisienne;

- sables du Flandrien de la région de Dunkerque;

- marnes et calcaires de Brie de la région parisienne ;

- argiles vertes de la région parisienne;

- argiles plastiques de la région parisienne;

- argiles des Flandres de la région de Dunkerque:

- marnes blanches de Pantin de la région parisienne ;

- marnes bleues d'Argenteuil de la région parisienne ;

- craie de la région parisienne.

\subsection{Etude d'histogrammes}

Avant toute recherche de corrélation, nous avons procédé à une étude des histogrammes des paramètres concernés, $\mathrm{pl}, \mathrm{E}$ et $\mathrm{N}$, afin de juger de la dispersion des résultats pour une même formation et par voie de conséquence de la fiabilité d'un essai par rapport à un autre; pour ces histogrammes, la situation idéale est celle qui se rapprocherait le plus de la distribution aléatoire de GAUSS.

Nous donnons à titre d'exemple les graphiques correspondants à la formation des argiles vertes du Sannoisien de la région parisienne, figures 10,11 et 12 , établis à la même échelle avec un pas identique, ce qui permet de les comparer directement. Pour cette formation, nous voyons immédiatement:

- que l'histogramme de la pression limite se rapproche le plus de la situation idéale;

- que celui du module pressiométrique s'en éloigne le plus avec une grande dispersion des résultats :

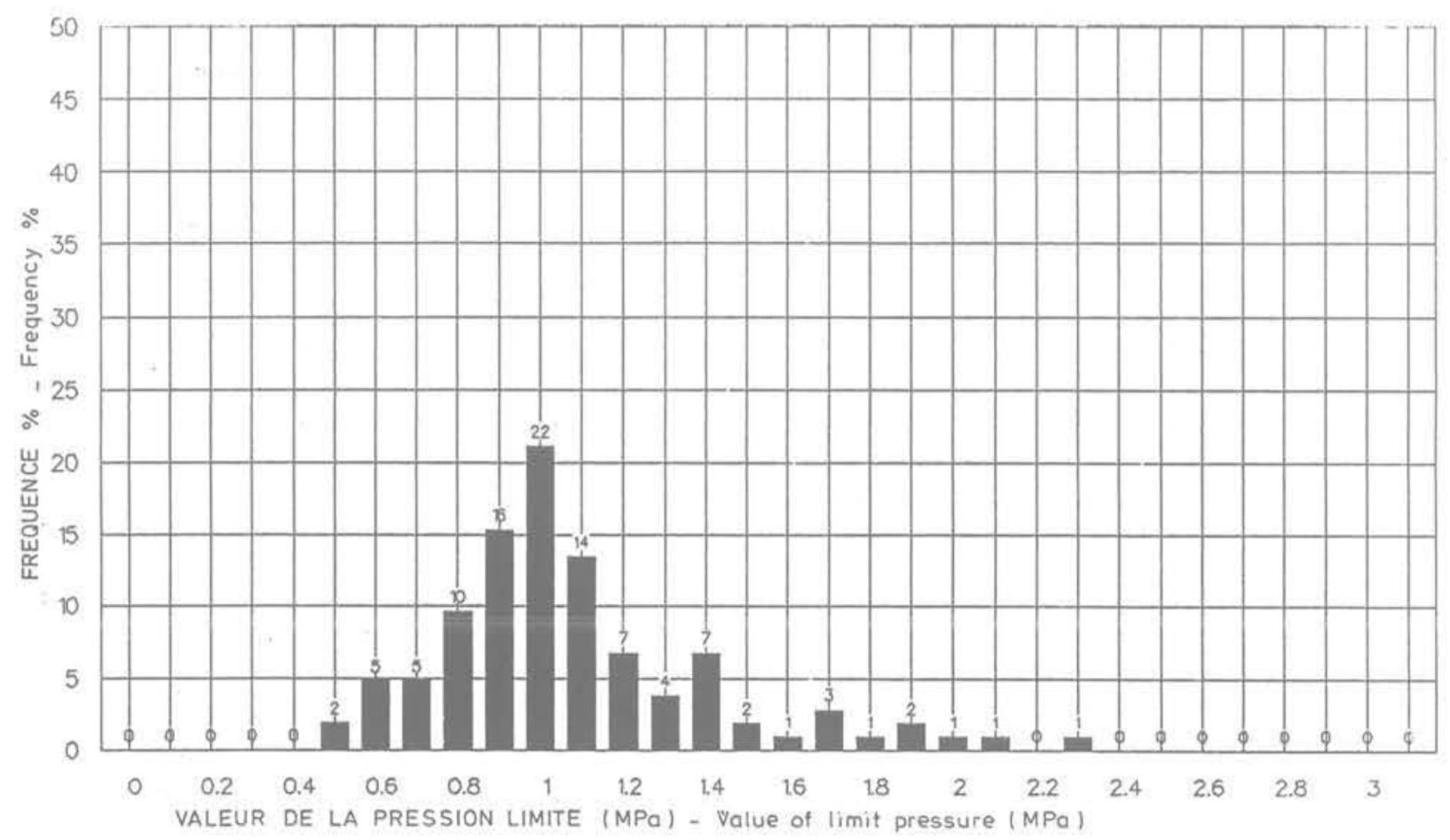

Fig. 10. - Argile verte de la région parisienne. 104 valeurs-histogramme des PI. Fig. 10. - Green clay of the Paris area. 104 values-PI histogram. 


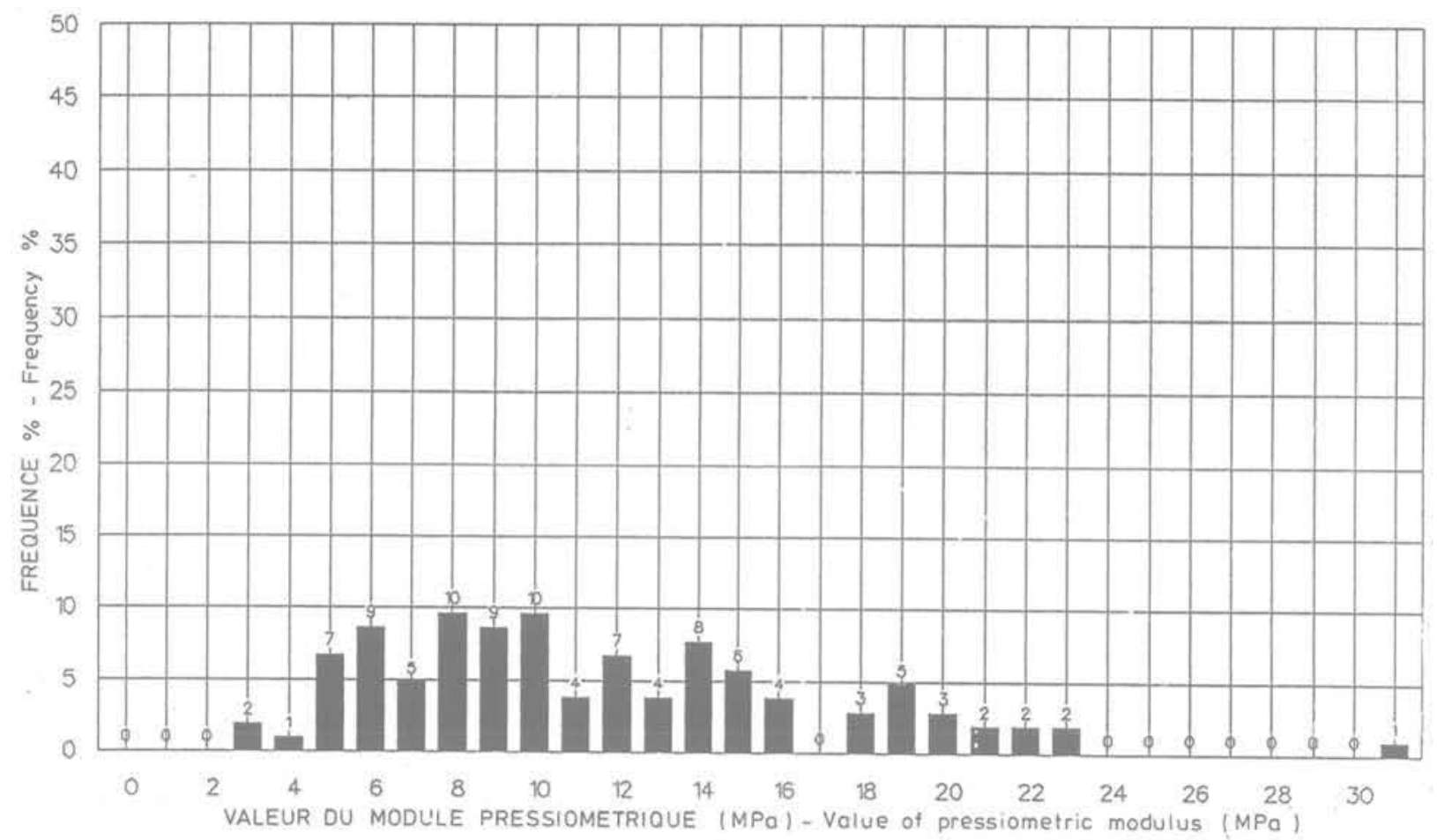

Fig. 11. - Argile verte de la région parisienne, 104 valeurs-histogramme des $E_{m}$. Fig. 11. - Green clay of the Paris area. 104 values $-E_{m}$ histogram.

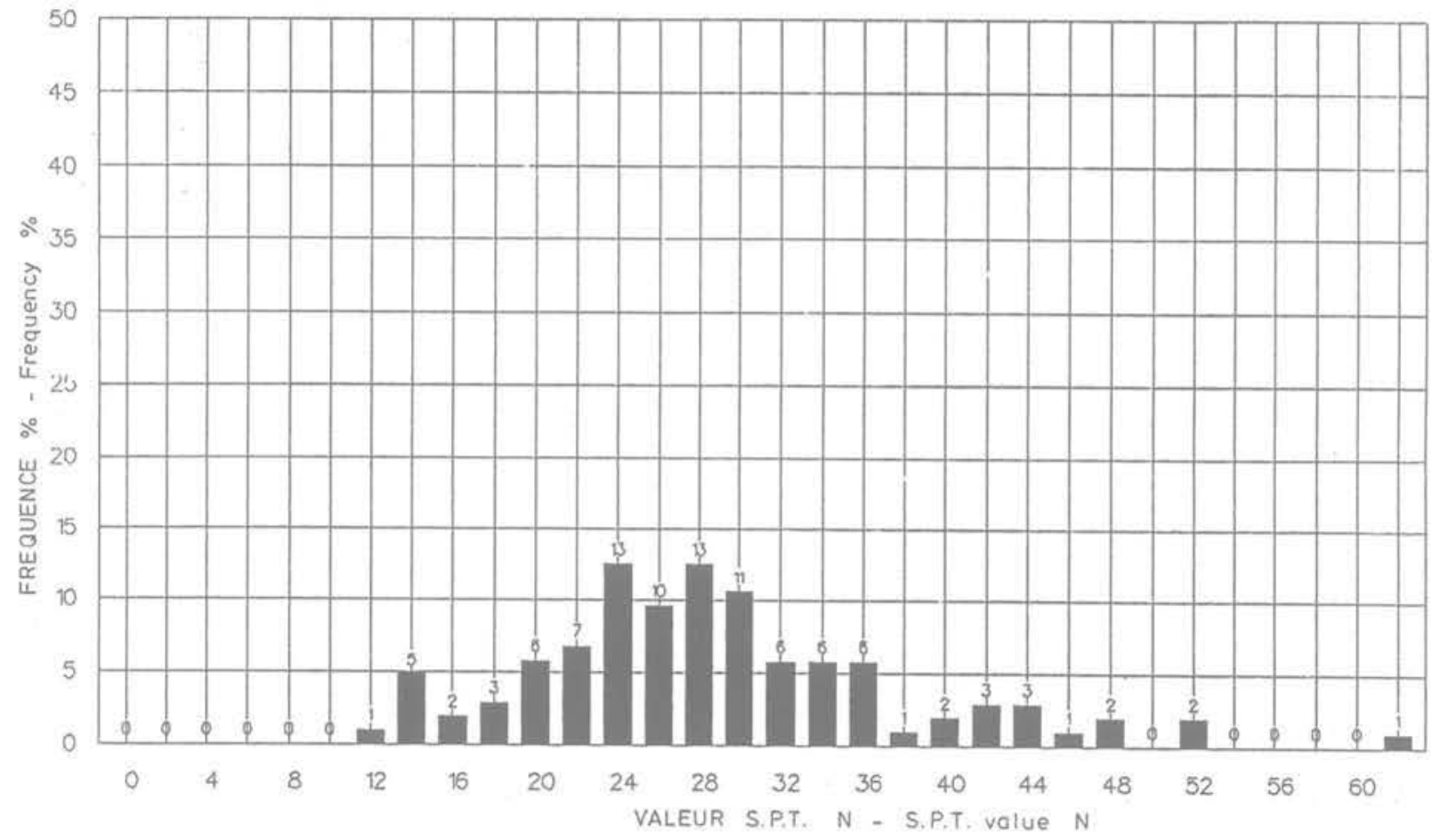

Fig. 12. - Argile verte de la région parisienne. 104 valeurs-histogramme des valeurs $N$. Fig. 12. - Green clay of Paris area. 104 values-N histogram.

- que l'histogramme de la valeur SPT est tout à fait satisfaisant avec près de $52 \%$ des valeurs regroupées entre 22 et 30 .

Cette constatation est générale et laisse présager que les corrélations entre $\mathrm{N}$ et $\mathrm{p} 1$ seront meilleures que celles entre $\mathrm{N}$ et $\mathrm{E}$.
Nous voyons par conséquent que les valeurs SPT sont tout aussi exploitables que les valeurs pressiométriques, bien qu'il s'agisse d'une formation argileuse ; il n'y a en fait aucune justification objective à ce que la validité de l'essai SPT soit exclusivement limitée aux sables. 


\subsection{Choix de la méthode statistique}

Afin de simplifier l'utilisation des corrélations, elles ont été recherchées sous la forme d'une équation de droite passant par l'origine. C'est pourquoi les corrélations trouvées sont des droites d'approximation calculées sur un ensemble de points par la méthode des moindres carrés en imposant une ordonnée nulle à l'origine.

La principale difficulté, comme pour toute étude statistique, consiste à trouver un algorithme qui permette de retirer quelques mesures, tout en conservant un nombre significatif de couples $(\mathrm{pl}, \mathrm{N})$ et $(\mathrm{E}, \mathrm{N})$ pour le calcul des droites d'approximation.

Cinq méthodes différentes ont été testées sur l'ensemble des mesures effectuées dans les limons bruns des plateaux de la région parisienne. Celle qui, tout en retirant un minimum de points (moins de $20 \%$, permet de trouver un pourcentage d'incertitude relativement correct ( $30 \%$ environ) a été retenue, et appliquée pour chaque formation géologique étudiée.

Phase préliminaire : tout d'abord, le premier travail consiste à former les couples $(\mathrm{pl}, \mathrm{N})$ et $(\mathrm{E}, \mathrm{N})$ des mesures pressiométriques et SPT dans les sondages les plus proches à une même profondeur et pour une même formation géologique.

Les couples ainsi formés sont placés sur un graphique avec en abscisse, pression limite ou module pressiométrique (exprimé en $\mathrm{MPa}$ ), en ordonnée, valeur $\mathrm{N}$ de l'essai de pénétration standard.

Quelques valeurs estimées trop éloignées de l'ensemble du nuage de points sont retirées. Le nombre de ces valeurs ne dépasse pas $10 \%$ du nombre initial.
Elles correspondent à des mesures aberrantes ou à des essais se rapportant à des sols non comparables. Phase 1 : les droites d'ajustement de $\mathrm{N}$ en fonction de $\mathrm{pl}$ et de $\mathrm{pl}$ en fonction de $\mathrm{N}$ sont calculées sur l'ensemble des points restants, les coefficients en étant déterminés par la méthode des moindres carrés. Il est admis de ne conserver que les points situés à l'intérieur des bandes de largeur $60 \%$ centrées sur ces droites.

Phase 2 : les droites d'ajustement passant par l'origine sont alors calculées, pour les couples restant; elles représentent les corrélations retenues pour la formation géologique ainsi étudiée.

La figure 13 représente, déterminé dans ces conditions, le segment de droite donnant la corrélation entre $\mathrm{pl}$ et $\mathrm{N}$ pour les argiles vertes de la région parisienne. La même démarche permet la corrélation entre $\mathrm{E}$ et $\mathrm{N}$, dont nous avons vu que la précision était moins satisfaisante que celle corrélant pl et $\mathrm{N}$ du fait d'une plus grande dispersion de $\mathrm{E}$.

Nous avons déterminé également une valeur caractérisant le pourcentage de dispersion des valeurs mesurées par rapport à la droite d'ajustement prise en compte pour la corrélation.

\section{RÉSULTATS}

Pour les 9 formations géologiques retenues, nous résumons dans le tableau II le nombre de mesures effectuées, ainsi que le nombre de couples formés à partir de ces mesures et celui des couples retenus. Nous voyons que les droites d'ajustement ont été déterminées à partir de l'analyse de 25 à 40 couples

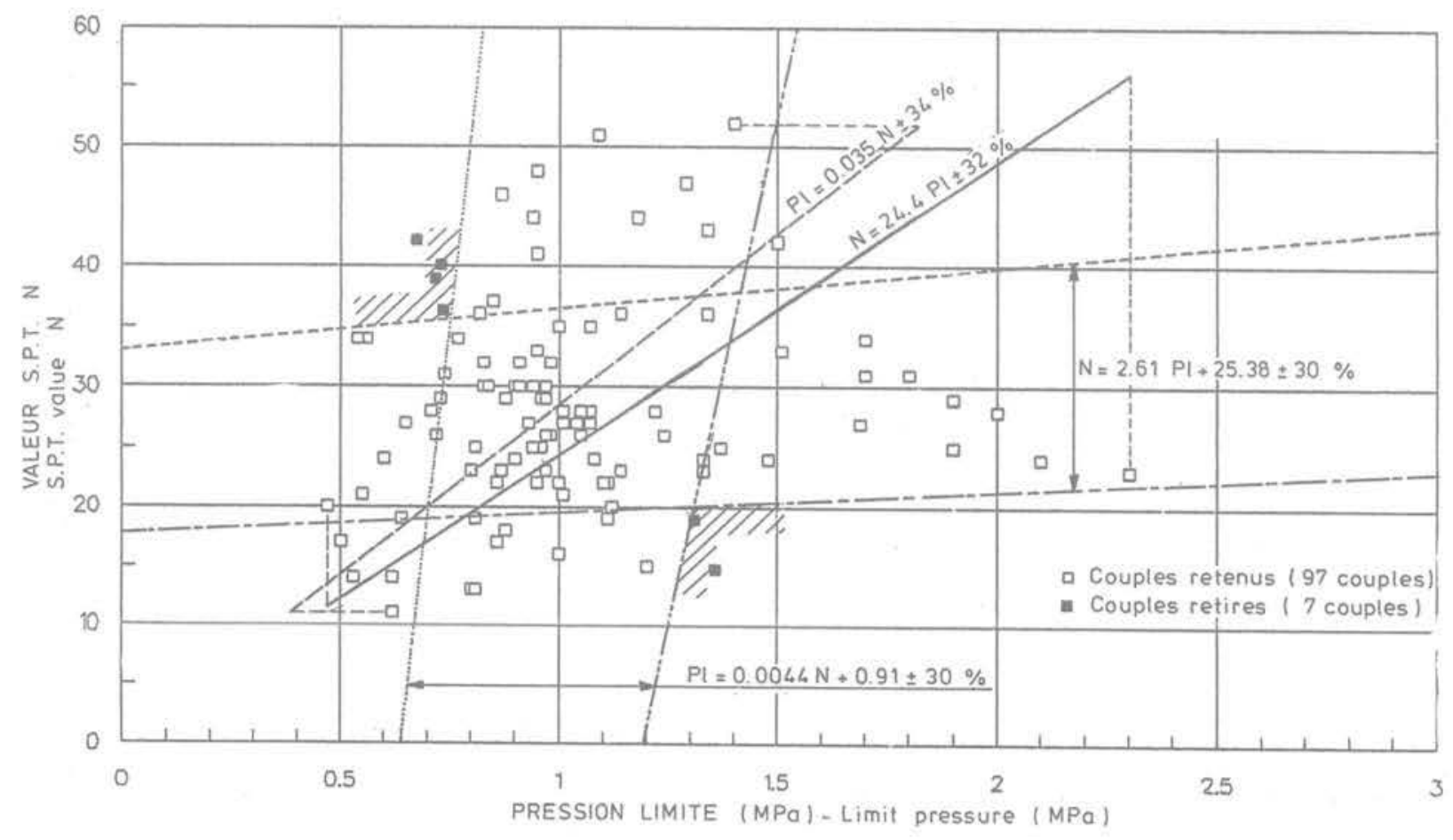

Fig. 13. - Argile verte de la région parisienne. Etude de corrélation sur 97 couples de valeurs. Fig. 13. - Green clay of Paris area. Correlation study for 97 couples of values. 
retenus par formation, à l'exception des argiles vertes pour lesquelles ce nombre est de 98.

Nous donnons pour chacune de ces formations les corrélations obtenues et les relations simplifiées pou. vant être utilisées avec les pourcentages d'incertitude et les plages d'utilisation (unités retenues $\mathrm{MPa}$ ).
L'examen du tableau III permet de faire les quelques remarques suivantes.

En premier lieu, on constate bien que le pourcentage d'incertitude défini, qui représente, en quelque sorte, l'ouverture du fuseau dans lequel se situent les points utilisés pour la corrélation, est moindre pour les cor-

Tableau II. - Nombre de couples de mesure ayant servi de base à l'étude.

Table II. - Number of couple of measures used for study.

\begin{tabular}{|c|c|c|c|c|c|c|c|c|c|}
\hline \multirow{2}{*}{$\begin{array}{c}\text { Désignation } \\
\text { des formations étudiées }\end{array}$} & \multicolumn{3}{|c|}{ Nombre de mesures } & \multicolumn{2}{|c|}{ Couples formés } & \multicolumn{4}{|c|}{ Couples retenus } \\
\hline & Total & Pressio. & SPT & $\mathrm{PI} / \mathrm{N}$ & $E / N$ & $\mathrm{Pl} / \mathrm{N}$ & $\%$ & $E / N$ & $\%$ \\
\hline Limons des plateaux & 137 & 72 & 65 & 53 & 53 & 43 & 78 & 41 & 73 \\
\hline Sables du Flandrien & 110 & 55 & 55 & 33 & 48 & 24 & 73 & 39 & 81 \\
\hline Marnes et calcaires de Brie & 120 & 64 & 56 & 37 & 41 & 31 & 84 & 28 & 68 \\
\hline Argiles vertes & 287 & 139 & 148 & 104 & 104 & 97 & 93 & 97 & 93 \\
\hline Argiles plastiques & 102 & 71 & 31 & 42 & 42 & 39 & 93 & 40 & 95 \\
\hline Argiles de Flandres & 44 & 22 & 22 & 22 & 22 & 21 & 95 & 21 & 95 \\
\hline Marnes de Pantin & 92 & 54 & 38 & 32 & 33 & 25 & 78 & 26 & 79 \\
\hline Marnes d'Argenteuil & 104 & 53 & 51 & 40 & 41 & 38 & 95 & 32 & 78 \\
\hline Craie & 125 & 48 & 77 & 38 & 45 & 32 & 84 & 36 & 80 \\
\hline
\end{tabular}

Tableau III. - Corrélations obtenues dans différentes formations.

Table III. - Correlation for different formations.

\begin{tabular}{|c|c|c|c|c|}
\hline Désignation des formations & Corrélations & $\%$ d'incertitudes & Plage d'utilisation & Formules simplifiées \\
\hline $\begin{array}{l}\text { Limons bruns des plateaux } \\
\text { région parisienne }\end{array}$ & $\begin{array}{l}\mathrm{N}=29,4 \mathrm{PI} \\
\mathrm{PI}=0,029 \mathrm{~N} \\
\mathrm{~N}=2,42 \mathrm{E} \\
\mathrm{E}=0,35 \mathrm{~N}\end{array}$ & $\begin{array}{l}34 \\
29 \\
35 \\
36\end{array}$ & $\begin{array}{l}0,1-0,72(P I) \\
0,1-32(N) \\
0,5-9 \text { (E) } \\
1-32(N)\end{array}$ & $\begin{array}{l}N=32 \mathrm{PI} \\
N=2,6 \mathrm{E}\end{array}$ \\
\hline $\begin{array}{l}\text { Sables du Flandrien } \\
\text { région du Nord }\end{array}$ & $\begin{array}{l}\mathrm{N}=19,91 \mathrm{PI} \\
\mathrm{Pl}=0,046 \mathrm{~N} \\
\mathrm{~N}=2,69 \mathrm{E} \\
E=0,33 \mathrm{~N}\end{array}$ & $\begin{array}{l}23 \\
24 \\
29 \\
31\end{array}$ & $\begin{array}{l}0,5-2,7(P)) \\
10-81(N) \\
3-29(E) \\
10-95(N)\end{array}$ & $\begin{array}{l}\mathrm{N}=21 \mathrm{PI} \\
\mathrm{N}=2,9 \mathrm{E}\end{array}$ \\
\hline $\begin{array}{l}\text { Marnes et calcaires } \\
\text { de Brie } \\
\text { de la région parisienne }\end{array}$ & $\begin{array}{l}\mathrm{N}=19,7 \mathrm{PI} \\
\mathrm{PI}=0,043 \mathrm{~N} \\
\mathrm{~N}=2,09 \mathrm{E} \\
E=0,39 \mathrm{~N}\end{array}$ & $\begin{array}{l}42 \\
37 \\
47 \\
43\end{array}$ & $\begin{array}{l}0,4-2,40 \text { (PI) } \\
8-40 \text { (N) } \\
1,7-18 \text { (E) } \\
1,40(N)\end{array}$ & $\begin{array}{l}N=21 \mathrm{PI} \\
\mathrm{N}=2,3 \mathrm{E}\end{array}$ \\
\hline $\begin{array}{l}\text { Argiles vertes } \\
\text { région parisienne }\end{array}$ & $\begin{array}{l}\mathrm{N}=24,4 \mathrm{PI} \\
\mathrm{PI}=0,035 \mathrm{~N} \\
\mathrm{~N}=2,10 \mathrm{E} \\
E=0,39 \mathrm{~N}\end{array}$ & $\begin{array}{l}32 \\
34 \\
38 \\
41\end{array}$ & $\begin{array}{l}0,5-2,3(\mathrm{Pl}) \\
1-52(\mathrm{~N}) \\
2,1-22,5(\mathrm{E}) \\
11-52(\mathrm{~N})\end{array}$ & $\begin{array}{l}\mathrm{N}=26 \mathrm{PI} \\
\mathrm{N}=2,3 \mathrm{E}\end{array}$ \\
\hline $\begin{array}{l}\text { Argiles plastiques } \\
\text { région parisienne }\end{array}$ & $\begin{array}{l}N=17,6 \mathrm{PI} \\
P I=0,054 \mathrm{~N} \\
N=1,57 \mathrm{E} \\
E=0,61 \mathrm{~N}\end{array}$ & $\begin{array}{l}20 \\
20 \\
18 \\
19\end{array}$ & $\begin{array}{l}1,1-2,5(\mathrm{Pl}) \\
15-46 \text { (N) } \\
12,5-25,9 \text { (E) } \\
15-46(\mathrm{~N})\end{array}$ & $\begin{array}{l}N=18 \mathrm{PI} \\
N=1,6 \mathrm{E}\end{array}$ \\
\hline $\begin{array}{l}\text { Argile des Flandres } \\
\text { région parisienne }\end{array}$ & $\begin{array}{l}\mathrm{N}=14,9 \mathrm{PI} \\
\mathrm{PI}=0,066 \mathrm{~N} \\
\mathrm{~N}=0,62 \mathrm{E} \\
\mathrm{E}=1,51 \mathrm{~N}\end{array}$ & $\begin{array}{l}13 \\
12 \\
25 \\
24\end{array}$ & $\begin{array}{l}0,6-2,0(P) \\
10-31(N) \\
8-56(E) \\
10-31(N)\end{array}$ & $\begin{array}{l}N=15 \mathrm{PI} \\
N=0,6 \mathrm{E}\end{array}$ \\
\hline $\begin{array}{l}\text { Marnes de Pantin } \\
\text { région parisienne }\end{array}$ & $\begin{array}{l}\mathrm{N}=23,6 \mathrm{PI} \\
\mathrm{PI}=0,041 \mathrm{~N} \\
\mathrm{~N}=1,55 \mathrm{E} \\
\mathrm{E}=0,55 \mathrm{~N}\end{array}$ & $\begin{array}{l}18 \\
18 \\
50 \\
42\end{array}$ & $\begin{array}{l}0,4-1,7(\mathrm{PI}) \\
10-38(\mathrm{~N}) \\
2,4-34(\mathrm{E}) \\
10-55(\mathrm{~N})\end{array}$ & $\begin{array}{l}\mathrm{N}=24 \mathrm{PI} \\
\mathrm{N}=1.7 \mathrm{E}\end{array}$ \\
\hline $\begin{array}{l}\text { Marnes d'Argenteuil } \\
\text { région parisienne }\end{array}$ & $\begin{array}{l}\mathrm{N}=20,6 \mathrm{PI} \\
\mathrm{PI}=0,042 \mathrm{~N} \\
\mathrm{~N}=1,76 \mathrm{E} \\
\mathrm{E}=0,48 \mathrm{~N}\end{array}$ & $\begin{array}{l}34 \\
35 \\
40 \\
36\end{array}$ & $\begin{array}{l}0,4-2,2(P) \\
10-50 \text { (N) } \\
2,2-27 \text { (E) } \\
10-41 \text { (N) }\end{array}$ & $\begin{array}{l}\mathrm{N}=22 \mathrm{PI} \\
\mathrm{N}=1,9 \mathrm{E}\end{array}$ \\
\hline $\begin{array}{l}\text { Craie } \\
\text { région parisienne }\end{array}$ & $\begin{array}{l}\mathrm{N}=5,8 \mathrm{PI} \\
\mathrm{PI}=0,154 \mathrm{~N} \\
\mathrm{~N}=0,63 \mathrm{E} \\
\mathrm{E}=1,38 \mathrm{~N}\end{array}$ & $\begin{array}{l}30 \\
34 \\
32 \\
33\end{array}$ & $\begin{array}{l}1,1-4,8 \text { (PI) } \\
3-32 \text { (N) } \\
5,5-51,5 \text { (E) } \\
3-32(N)\end{array}$ & $\begin{array}{l}N=6 \mathrm{Pl} \\
N=0,7 \mathrm{E}\end{array}$ \\
\hline
\end{tabular}

$\mathrm{N}=$ valeur $\mathrm{SPT} ; \mathrm{PI}=$ Pression Limite $(\mathrm{MPa}) ; \mathrm{E}=$ Module pressiométrique $(\mathrm{MPa})$. 
rélations entre $\mathrm{N}$ et $\mathrm{Pl}$ (moyenne : $27 \%$, avec des valeurs allant de $12 \%$ - argiles du Nord - à $42 \%$ - marnes et calcaires de Brie) que pour les corrélations entre $\mathrm{N}$ et $\mathrm{E}$ (moyenne : $34 \%$ avec des valeurs allant de $18 \%$ - argiles plastiques de la région parisienne - à $50 \%$ - marnes de Pantin).

Par ailleurs, les meilleurs résultats sont obtenus dans les puissantes formations argileuses, alors que les formations au faciès moins constant donnent logiquement des résultats moins homogènes. Ceci montre bien que l'essai SPT donne, dans les argiles, des résultats aussi valables que l'essai pressiométrique, en terme de représentativité des échantillons de valeurs étudiés. Si on le compare au module MENARD de l'essai pressiométrique, il apparaît plus fiable.

En second lieu, la craie et l'argile ypresienne du Nord présentent deux cas singuliers. Pour la craie, cela confirme bien le comportement particulier de ce matériau. Pour l'argile ypresienne du Nord, on trouve logiquement une corrélation N-Pl comparable à celle obtenue pour les argiles plastiques de la région parisienne, avec un pourcentage d'incertitude remarquable $(12 \%)$, mais une corrélation $\mathrm{N}-\mathrm{E}$ très différente et unique pour les argiles étudiées $(\mathrm{N}=0,6 \mathrm{E})$. Cette dernière corrélation, qui correspond à un rapport $\mathrm{E} / \mathrm{PI}$ de 25 assez inhabituel, doit être considérée avec circonspection.

\section{CONCLUSIONS}

L'étude réalisée sur neuf formations géologiques caractéristiques de la région parisienne et du Nord de la France a permis d'établir des corrélations entre les résultats pressiométriques et SPT. Les figures 14 et 15 représentent les résultats à retenir entre la pression limite et la valeur SPT selon le type de terrain. Les différents segments de droite indiquent la plage des valeurs obtenues dans chacune des formations étudiées.

D'une manière simple, pour les cinq principaux types de sols meubles où pressiomètres et SPT sont utilisés, les relations à considérer entre les deux types d'essais sont données dans le tableau IV.

Tableau IV. - Corrélations simplifiées proposées entre $N, P I(\mathrm{MPa})$ et $E(\mathrm{MPa})$.

Table IV. - Simplified correlations proposed between N. Pl (MPa) and E (MPa)

\begin{tabular}{|l|l|l|}
\hline Nature du sol & $\begin{array}{c}\text { Corrélation } \\
\mathrm{PI} \rightarrow \mathrm{N}\end{array}$ & $\begin{array}{c}\text { Corrélation } \\
\mathrm{E}-\mathrm{N}\end{array}$ \\
\hline Limons & $\mathrm{N}=32 \times \mathrm{PI}$ & $\mathrm{N}=2,6 \mathrm{E}$ \\
Sables & $\mathrm{N}=21 \times \mathrm{PI}$ & $\mathrm{N}=2,9 \mathrm{E}$ \\
Argiles vertes & $\mathrm{N}=26 \times \mathrm{PI}$ & $\mathrm{N}=2,3 \mathrm{E}$ \\
Argiles plastiques & $\mathrm{N}=18 \times \mathrm{PI}$ & $\mathrm{N}=1,6 \mathrm{E}$ \\
Marnes & $\mathrm{N}=23 \times \mathrm{PI}$ & $\mathrm{N}=1,9 \mathrm{E}$ \\
Craie & $\mathrm{N}=6 \times \mathrm{PI}$ & $\mathrm{N}=0,7 \mathrm{E}$ \\
\hline
\end{tabular}

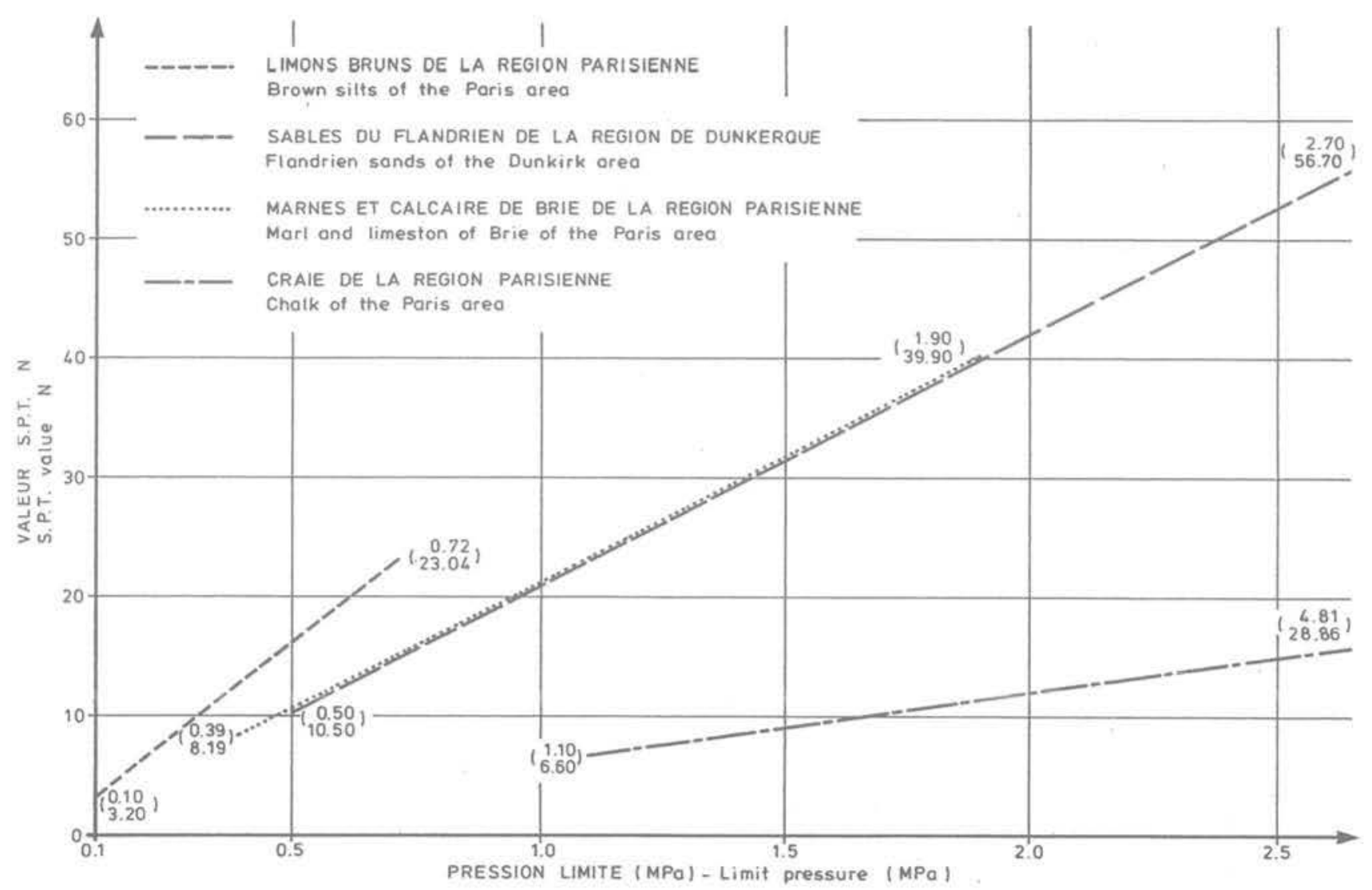

Fig. 14. - Corrélations SPT N-PI, limons, sable, marne, craie (Paris ou nord de la France).

Fig. 14. - Correlations SPT N values N.PI, silts, sand, marls, chalk (Paris or north of France). 


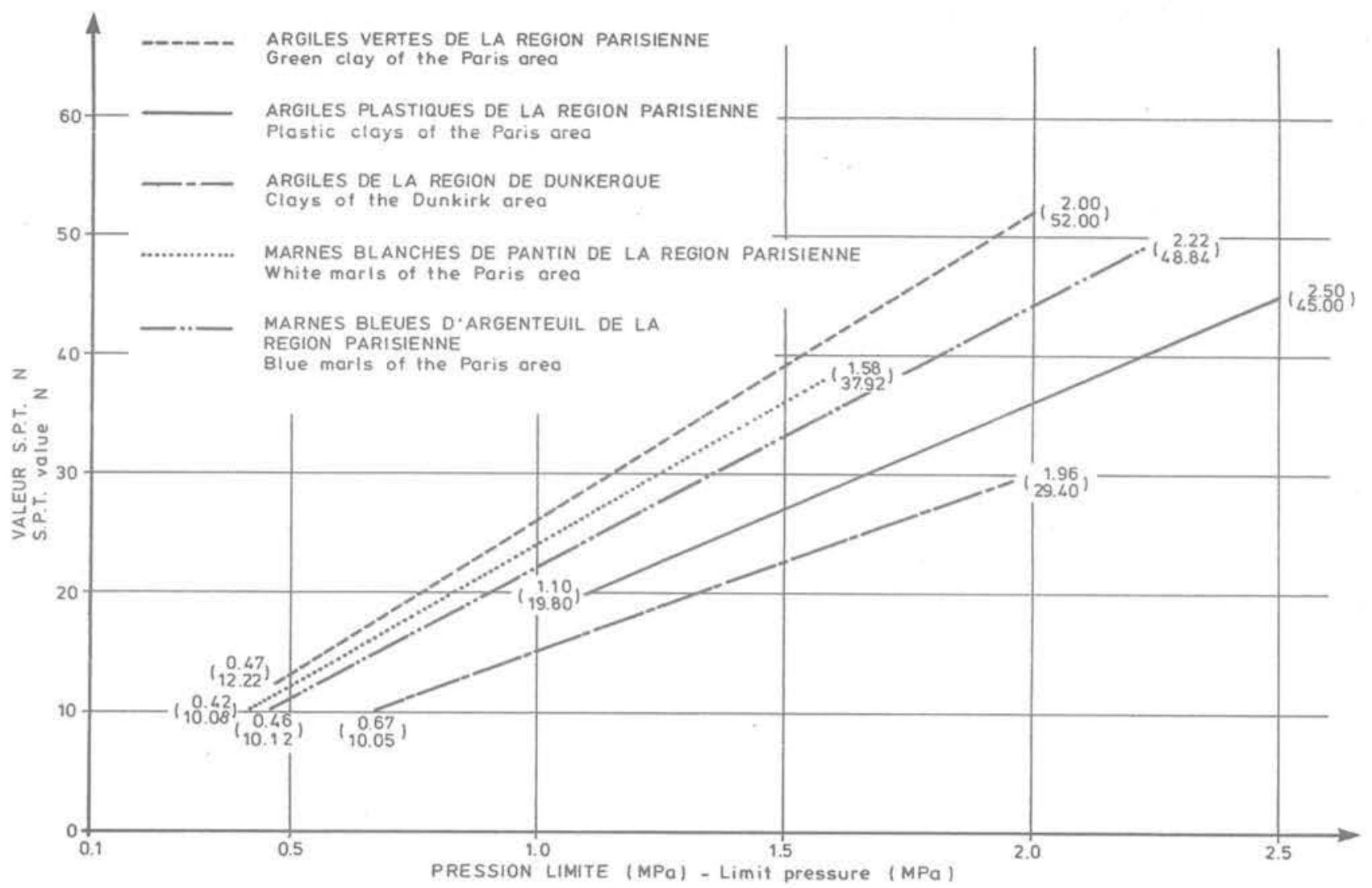

Fig. 15. - Corrélations SPT N-Pl, argiles et marnes argileuses (Paris ou nord de la France). Fig. 15. - Correlations SPT N-PI, clays and clayed marls (Paris or north of France).

Contrairement à certaines idées reçues, les corrélations entre les valeurs des essais SPT et pressiométriques sont tout à fait valables dans les argiles, et rien ne justifie d'accorder dans ces terrains, une préférence à un type d'essai plutôt qu'à l'autre.

La craie constitue un cas particulier; en effet, les essais de pénétration doivent être interprétés avec discernement dans cette formation, étant donné son caractère thixotropique.

Les corrélations ne doivent pas être utilisées en dehors du domaine d'application des essais concernés en particulier dans les terrains trop mous (vases) ou au contraire trop résistants (roches dures).

En revanche, cette étude montre que, quels que soit les terrains, pourvu qu'ils soient meubles (limons, sables, argiles, marnes, craie), il est possible de passer de la valeur SPT à la pression limite ou au module de déformation avec une approximation qui reste dans des limites acceptables, et cette correspondance permet donc d'utiliser les valeurs SPT pour donner une estimation des tassements par la méthode pressiométrique; les erreurs qui peuvent découler de l'utilisation judicieuse de corrélations ne sont pas plus importantes que celles qui proviennent de la détermination directe du module pressiométrique lui-même ou de la validité des formules utilisées. Le seul fait qu'il n'existe pas de relation théorique ou semiempirique directe reconnue entre les valeurs SPT et les tassements ne peut être de nature à déconseiller l'utilisation de cette méthode d'investigation, ainsi qu'ont parfois tendance à le suggérer, en France, les praticiens et les auteurs des recommandations ou normes techniques. Cela revient à dénigrer l'intérêt des corrélations, pourtant très largement utilisées par ailleurs en mécanique des sols.

Cette mise au point et cette conclusion justifient, en quelque sorte, la pratique mondiale extensive du SPT, quelque peu combattue, en France, au profit de l'essai pressiométrique, dont nous ne contestons pas, bien entendu, le grand intérêt.

\section{BIBLIOGRAPHIE}

CASSAN M. (1978), Les essais in situ en mécanique des sols, tome 1 réalisation et interprétation, Eyrolles, Paris.

CORTE J.-F. (1985), IV-5 L'évaluation du risque de liquéfaction à partir des essais en place, Génie parasismique, Presses ENCP, pp. 323-331. 
COVILLE-FEUILLADE M.-P. (1990), Corrélations entre le Standard Penetration Test et le pressiomètre, mémoire présenté en vue de lobtention du diplôme d'ingénieur en géophysique et géotechnique, le 19.10.90, Institut en Sciences et Technologie, université Pierre et Marie CURIE.
MEYNARD L. (1975), Notice générale D60, Le Pressiomètre, pp. 3-12.

SANGLERAT G. (1972), The penetrometer and soil exploration, Elsevier Publishing Compagny Amsterdam-London-New York, pp. 145-155. 